\title{
Indirect dynamics in a highly exoergic substitution
}

\section{reaction}

Jochen Mikosch, ${ }^{\dagger}$ Jiaxu Zhang, ${ }^{\ddagger}$ Sebastian Trippel, ${ }^{\text {II }}$ Christoph Eichhorn, ${ }^{\S}$ Rico

Otto,\|,@ Rui Sun, ${ }^{\ddagger}$ Wibe A. de Jong, ${ }^{\perp}$ Matthias Weidemüller, ${ }^{\#}$ William L. Hase, ${ }^{\ddagger}$ and Roland Wester*,\|

National Research Council of Canada, Ottawa, Ontario K1A OR6, Canada, Department of Chemistry and Biochemistry, Texas Tech University, Lubbock, Texas 79409-1061, USA, Center for Free-Electron Laser Science, DESY, Notkestraße 85, 22607 Hamburg, Germany, Physikalisches Institut, Universität Freiburg, Hermann-Herder-Straße 3, 79104 Freiburg, Germany, Institut für Ionenphysik und Angewandte Physik, Universität Innsbruck, Technikerstraße 25/3, 6020 Innsbruck, Austria, EMSL, Pacific Northwest National Laboratory, P.O. Box 999, Richland, Washington 99352, USA, and Physikalisches Institut, Universität Heidelberg, Philosophenweg 12, 69120 Heidelberg, Germany

E-mail: roland.wester@uibk.ac.at

\footnotetext{
${ }^{*}$ To whom correspondence should be addressed

${ }^{\dagger}$ National Research Council of Canada, Ottawa, Ontario K1A 0R6, Canada

¥Department of Chemistry and Biochemistry, Texas Tech University, Lubbock, Texas 79409-1061, USA

${ }^{\mathbb{I}}$ Center for Free-Electron Laser Science, DESY, Notkestraße 85, 22607 Hamburg, Germany

${ }^{\S}$ Physikalisches Institut, Universität Freiburg, Hermann-Herder-Straße 3, 79104 Freiburg, Germany

"Institut für Ionenphysik und Angewandte Physik, Universität Innsbruck, Technikerstraße 25/3, 6020 Innsbruck,

${ }^{\perp}$ EMSL, Pacific Northwest National Laboratory, P.O. Box 999, Richland, Washington 99352, USA

\#Physikalisches Institut, Universität Heidelberg, Philosophenweg 12, 69120 Heidelberg, Germany

${ }^{\circledR}$ Department of Chemistry and Biochemistry, University of California, San Diego, 9500 Gilman Drive, La Jolla, 92093-0340, CA, USA
} Austria 


\begin{abstract}
The highly exoergic nucleophilic substitution reaction $\mathrm{F}^{-}+\mathrm{CH}_{3} \mathrm{I}$ shows strikingly different reaction dynamics than substitution reactions of larger halogen anions. Over a wide range of collision energies, a large fraction of indirect scattering via a long-lived hydrogenbonded complex is found both in crossed-beam imaging experiments and in direct chemical dynamics simulations. Our measured differential scattering cross sections show large-angle scattering and low product velocities for all collision energies, resulting from efficient transfer of the collision energy to internal energy of the $\mathrm{CH}_{3} \mathrm{~F}$ reaction product. Both findings are in strong contrast to the previously studied substitution reaction of $\mathrm{Cl}^{-}+\mathrm{CH}_{3} \mathrm{I}$ [Science $\mathbf{2 0 0 8}$, $319,183-186]$ at all but the lowest collision energies, a discrepancy that was not captured in a subsequent study at only a low collision energy [J. Phys. Chem. Lett. 2010, 1, 2747-2752]. Our direct chemical dynamics simulations at the DFT/B97-1 level of theory show that the reaction is dominated by three atomic-level mechanisms, an indirect reaction proceeding via an $\mathrm{F}^{-}-\mathrm{HCH}_{2} \mathrm{I}$ hydrogen-bonded complex, a direct rebound and a direct stripping reaction. The indirect mechanism is found to contribute about half of the overall substitution reaction rate at both low and high collision energies. This large fraction of indirect scattering at high collision energy is particularly surprising, since the barrier for the $\mathrm{F}^{-}-\mathrm{HCH}_{2} \mathrm{I}$ complex to form products is only $0.10 \mathrm{eV}$. Overall, experiment and simulation agree very favorably in both the scattering angle and the product internal energy distributions.
\end{abstract}

\title{
Introduction
}

Bimolecular nucleophilic substitution $\left(\mathrm{S}_{\mathrm{N}} 2\right)$ reactions

$$
\mathrm{X}^{-}+\mathrm{CH}_{3} \mathrm{Y} \longrightarrow \mathrm{CH}_{3} \mathrm{X}+\mathrm{Y}^{-}
$$

are among the most simple and most prevalent yet most multifaceted ion-molecule reactions. Therefore - and due to their fundamental importance in organic chemistry ${ }^{1}$ - they have become extensively studied model systems in physical chemistry and chemical physics both experimen- 
tally $^{2-4}$ and computationally. ${ }^{5-7}$ During the last few years it has become possible to directly observe the dynamics of ion-molecule reactions by kinematically complete imaging of reactive scattering, which has been applied to charge transfer reactions ${ }^{8,9}$ and to nucleophilic substitution reactions. ${ }^{10-12}$

The richness of gas-phase $S_{N} 2$ reactions is due to a characteristic potential barrier along the usually co-linear reaction path of reaction (1), which hinders the formation of reaction products. ${ }^{13}$ The barrier corresponds to an inversion at the carbon atom in Eq. (1) and separates the entrance and exit channel ion-dipole complex, thus forming a prototypical double-minimum potential energy landscape. ${ }^{14}$ Thermal rate coefficients for $\mathrm{S}_{\mathrm{N}} 2$ reactions can differ by several orders of magnitude and are usually far below the capture limit. ${ }^{15-18}$ This low reaction efficiency is ascribed to the low efficiency for crossing the central barrier. In the dynamical description of $\mathrm{S}_{\mathrm{N}} 2$ reactions of small reactants, the barrier represents a severe hindrance to product formation even if it is submerged with respect to the reactant energy asymptote. This is found to be caused by the inefficiency of intermolecular to intramolecular energy transfer necessary to surmount the barrier and break the $\mathrm{CH}_{3}-\mathrm{Y}$ bond. ${ }^{5}$ The transition states and thereby the reaction rate constants are also strongly affected by different substituents. ${ }^{4}$

In the statistical description of $\mathrm{S}_{\mathrm{N}} 2$ reactions the small rate for barrier crossings is explained by the high rotational (centrifugal) energy and low density of states at the barrier, which represents a transition state for product formation. The conditions for the applicability of statistical models for energy redistribution during $\mathrm{S}_{\mathrm{N}} 2$ reactions are subject of a longstanding debate. ${ }^{19-25}$ Statistical theories are found to be successful for larger systems as well as for decreasing temperature, where reaction rates are found to increase strongly. ${ }^{26,27}$ This is traced back to the fact that the most efficient way of crossing the central barrier is the formation of a long-lived entrance channel iondipole complex in which energy can be redistributed. As recently confirmed experimentally, the lifetime of the reaction intermediate increases with decreasing temperature. ${ }^{28}$ This leaves more time for transfer of energy to the critical modes, which drive the system over the barrier and lead to formation of reaction products. For the same reason molecular reaction products emerging from 
such a complex-mediated reaction pathway usually feature significant internal excitation. ${ }^{10,24}$

It has been established by both experiment and theory for reactions of chlorine anions with the methyl halide molecules $\mathrm{CH}_{3} \mathrm{Br}$ and $\mathrm{CH}_{3} \mathrm{I}$ that for collision energies above a few hundred meV direct reaction mechanisms dominate the dynamics, whereas for low collision energies complexmediated dynamics occur. ${ }^{10,24} \mathrm{~A}$ similar result has been reported for $\mathrm{F}^{-}$reacting with $\mathrm{CH}_{3} \mathrm{Cl}^{29}$ In a previous article we showed results for the nucleophilic substitution reaction

$$
\mathrm{F}^{-}+\mathrm{CH}_{3} \mathrm{I} \longrightarrow \mathrm{CH}_{3} \mathrm{~F}+\mathrm{I}^{-} \quad(\Delta \mathrm{H}=-1.84 \mathrm{eV}) .
$$

at a low collision energy of $0.32 \mathrm{eV}$ that were in agreement with this supposition. ${ }^{11}$ Specifically, we found that the reaction occurs by three different reaction mechanisms, the most probable one being the indirect, complex-mediated mechanism. ${ }^{11}$ The reaction is the most exothermic one of all halogen-methyl halide $S_{N}$ 2-reactions with a central barrier of only $0.1 \mathrm{eV}$ with respect to the entrance channel complex. ${ }^{30}$ This is suggestive of fast and direct dynamics and is supported by the high reaction probability of this system. ${ }^{31}$

Interestingly, the structure of the entrance channel complex of reaction (2) is not co-linear with $C_{3 v}$ symmetry, as for most halogen-methyl halide $\mathrm{S}_{\mathrm{N}} 2$-reactions, but is instead of $C_{s}$ symmetry ${ }^{30}$ with the fluorine anion attached to one of the three hydrogen atoms. The intrinisic reaction coordinate potential energy curve for the $\mathrm{F}^{-}+\mathrm{CH}_{3} \mathrm{I} \rightarrow \mathrm{CH}_{3} \mathrm{~F}+\mathrm{I}^{-}$reaction has been discussed previously ${ }^{30}$ and is plotted in Fig. 1. Shown for comparison is the potential energy curve for the reactive system constrained to a $\mathrm{C}_{3 v}$ geometry as for a traditional $\mathrm{S}_{\mathrm{N}} 2$ potential energy surface, ${ }^{2}$ which connects the reactants with the post-reaction ion-dipole complex without an intermediate energy barrier. To study the effect of hydrogen-bonding in ion-molecule complexes, infrared spectroscopy has been applied to halide anion-water clusters, which can be viewed as trapped entrance channel intermediates in the respective bimolecular reaction. ${ }^{32,33}$ As a function of proton affinity of the anion, a transition from a relatively weak ion-molecule interaction to a near covalent bond, where the proton is shared between the two centers, was established. ${ }^{34}$ 
Here we investigate reaction (2) for several different collision energies in the range where the transition from complex-mediated to direct reaction dynamics is expected. Our combined experimental-theoretical study employs ion imaging of crossed-beam reactive scattering ${ }^{10}$ and chemical dynamics simulations. ${ }^{35,36}$ We find experimentally that upon increasing the collision energy most of the energy available from reactant translation and reaction exothermicity is transferred into internal degrees of freedom of the molecular reaction product $\mathrm{CH}_{3} \mathrm{~F}$ - in strong contrast to our previous findings for the $\mathrm{Cl}^{-}+\mathrm{CH}_{3} \mathrm{I}$ reaction system. ${ }^{10}$ This evidence for indirect reaction mechanisms is supported by a very weak dependence of the cross section on the scattering angle. Both the energy- and the angle-resolved data are in very good agreement with the chemical dynamics simulations. The simulations find several reaction mechanisms to contribute, with the indirect complex-mediated mechanism being very important both at low and high collision energies. Together with the recently discovered "roaming" 37,38 and "roundabout" 10 mechanisms, this shows that indirect mechanisms in general can not be neglected at high collision energies in chemical reaction dynamics.

\section{Experimental procedure}

In the following we present the crossed beam imaging setup, the data acquisition system and the subsequent data reduction and analysis, which have not yet been described in detail. We also evaluate the attainable accuracy for the product velocities and the product internal energies measured in the crossed-beam imaging experiments.

\section{Data acquisition}

Similar to our previous experiment on $\mathrm{Cl}^{-}+\mathrm{CH}_{3} \mathrm{I},{ }^{10}$ scattering experiments have been performed with a pulsed ion beam of $\mathrm{F}^{-}$and a neutral beam of $\mathrm{CH}_{3} \mathrm{I}$ that are crossed inside a velocity map imaging spectrometer. $\mathrm{I}^{-}$reaction products are imaged onto a position sensitive detector that records their transverse velocity vectors in the scattering plane by using time-slicing. 
Ion and neutral reactant beams are provided by two differentially pumped source chambers and cross at $120^{\circ}$ in the lab frame. Both sources employ a pulsed supersonic expansion exiting from a piezo-electric valve built in-house, which is based on an older construction by $\mathrm{D}$. Gerlich and co-workers. In the ion source we seed the precursor $\mathrm{NF}_{3}$ at $10 \%$ in argon and ionize the gas mixture close to the nozzle by a short pulse of free electrons with about $100 \mathrm{eV}$ kinetic energy. $\mathrm{F}^{-}$reactants are then created very efficiently by dissociative attachment of secondary electrons and form a short packet. The velocity distribution of the prepared $\mathrm{F}^{-}$ion packet is imaged with the velocity map imaging spectrometer. It yields an energy-tunable reactant beam with a kinetic energy width of $0.2 \mathrm{eV}$ (FWHM) for mean kinetic energies between 0.2 and about $2 \mathrm{eV}$ and a typical angular spread of $15^{\circ}$ (FWHM).

The neutral source provides $\mathrm{CH}_{3} \mathrm{I}$ seeded at $10 \%$ in a pulsed supersonic expansion of helium. Special care has been taken to avoid clustering in the expansion, which we diagnose by photodissociation with a pulsed dye laser. ${ }^{39}$ Hence, we operated the expansion source at a stagnation pressure of only 0.8 bar, with the nozzle heated to $363 \mathrm{~K}$. This keeps the fraction of clustered reactants negligible. The $\mathrm{CH}_{3} \mathrm{I}$ velocity distribution is probed by imaging $\mathrm{CH}_{n} \mathrm{I}^{+}(n \leq 3)$ produced by electron impact ionization in the interaction region. We measure an absolute beam velocity of $890 \mathrm{~m} / \mathrm{s}$ for the helium/methyl iodide mixture with velocity spreads of around $60 \mathrm{~m} / \mathrm{s}$ (r.m.s.) in the parallel and $30 \mathrm{~m} / \mathrm{s}$ (r.m.s.) in the perpendicular direction. This corresponds to a translational temperature of around $60 \mathrm{~K}$. This temperature also gives a proxy of the $\mathrm{CH}_{3} \mathrm{I}$ rotational temperature. It is higher than in other molecular beam experiments with this molecule, because of the employed low stagnation pressure used to avoid clustering. Due to the inefficiency of vibrational cooling and the high seeding fraction the vibrational temperature of $\mathrm{CH}_{3} \mathrm{I}$ might be considerably higher than $60 \mathrm{~K}$, where an upper bound is given by the temperature of the nozzle $(363 \mathrm{~K})$.

The relative collision energy is tuned by changing the potential of the ion source relative to the field-free interaction region, which adjusts the mean velocity of the $\mathrm{F}^{-}$reactant beam. To ensure stable conditions, we measure its velocity distribution and arrival time in the interaction region with the pulsed-field imaging spectrometer before and after the acquisition of scattering data. 
Once the two reactant beams are synchronized in the initially field-free interaction region of the spectrometer, $\mathrm{I}^{-}$product ions emerge from reactive collisions. We also found proton transfer (with $\mathrm{CH}_{2} \mathrm{I}^{-}$products) and dihalde formation (with $\mathrm{IF}^{-}$products) to occur in collisions of $\mathrm{F}^{-}$with $\mathrm{CH}_{3} \mathrm{I}$. The dynamics observed for these channels will be described in an upcoming publication. The $\mathrm{I}^{-}$ product ions are mapped onto the position sensitive detector by a rapidly switched extraction field applied 3-5 $\mu$ s after the first reactions occurred. To suppress broadening of the images due to the finite size of the interaction volume we implement velocity mapping ${ }^{40}$ with a multi-stage extraction field, which allows for a more homogeneous electric potential in the interaction volume. We then employ time-slice imaging ${ }^{41-43}$ by gating the detection and thereby limit it to product ions with velocity vectors approximately parallel to the detector surface, i.e. in the scattering plane. Based on the detector gate of below $20 \mathrm{~ns}$ and the arrival time dispersion of the product ions of about $100 \mathrm{~ns}$ per $300 \mathrm{~m} / \mathrm{s}$ in the vertical velocity component, we estimate that the out-of-plane velocity is restricted to below $\pm 30 \mathrm{~m} / \mathrm{s}$. After time-slicing, we observe typically 1-5 scattering events per bunch crossing in this experiment, benefiting from the synchronous detection of all scattering angles. The data presented here contains $0.2-2 \times 10^{6}$ scattering events for each collision energy, acquired during time periods of typically $12 \mathrm{~h}$ at a repetition rate of the bunch crossing of $10 \mathrm{~Hz}$.

\section{Data analysis}

The product ion impact position $\vec{r}=(x, y)$ on the detector is converted into a laboratory velocity via

$$
\vec{v}_{\text {lab }}^{\prime}=\frac{\vec{r}-\vec{r}_{0}}{M t_{\text {flight }}} .
$$

The zero-velocity origin point $\vec{r}_{0}$ is determined via electron impact ionization of xenon, which can be leaked into the chamber and thermalizes with its walls at room temperature. The ion flight time from the interaction region to the detector $t_{\text {flight }}$ is defined by the sliced detection, and the magnification of the imaging lens is derived as $M=1.03$ from ion trajectory simulations. ${ }^{44}$

Reactive scattering with randomly oriented reactants is cylindrically symmetric around the 
relative velocity axis of the reactants, which yields up-down symmetry in the obtained images with respect to $\vec{v}_{\text {rel }}$. Experimentally, an asymmetry is introduced for higher ion velocities, which is caused by the loss of fast product ions from the collection volume of the spectrometer before the extraction field is pulsed on. This loss is described by an angle-independent, monotonically decreasing function of the magnitude of the lab velocity. The global probability loss function $P\left(\left|\vec{v}_{\text {lab }}^{\prime}\right|\right)$ is constructed for all data sets from the laboratory velocity image measured at $2.34 \mathrm{eV}$, by comparing image entries that are symmetrically positioned with respect to the relative velocity axis.

The linear transformation $\vec{v}_{\text {lab }}^{\prime}-\vec{v}^{\mathrm{cm}}$ yields the product velocity for scattering event in the center-of-mass frame ( $\vec{v}^{\mathrm{cm}}$ is the velocity of the center-of-mass in the laboratory frame). A rotation is then applied to align the relative velocity vector $\vec{v}_{\text {rel }}$ along the x-axis. The such transformed and rotated scattering events in the center-of-mass frame $\vec{v}_{\mathrm{cm}}^{\prime}$ are filled with their respective weight $P\left(\left|\vec{v}_{\text {lab }}^{\prime}\right|\right)^{-1}$ into a two-dimensional histogram that represents the differential scattering cross section

$$
\frac{d^{3} \sigma}{d v_{x} d v_{y} d v_{z}}=\frac{1}{v^{2}} \frac{d \sigma}{d v d \Omega}
$$

The representation on the left side of the equation is most instructive since an isotropic and homogeneous distribution of scattering products shows up as a uniform disposal in these histograms.

From the velocities in the center-of-mass frame, the scattering angle $\theta$ (defined as the angle between the initial $\mathrm{CH}_{3} \mathrm{I}$ and the final $\mathrm{I}^{-}$velocity vectors, see the Newton diagram in Fig. 2) and the internal energy $E_{\text {int }}$ of the products are calculated for each scattering event. $E_{\text {int }}$ is obtained as the total available energy minus the kinetic energy of the products $E_{\text {int }}^{\mathrm{CH}_{3} \mathrm{~F}}=\left(E_{\text {rel }}-\Delta H\right)-E_{\text {rel }}^{\text {products }}$. It equals the internal excitation energy of the $\mathrm{CH}_{3} \mathrm{~F}$ product, since the ionic product $\mathrm{I}^{-}$has no stable excited states. Internal excitation of the $\mathrm{CH}_{3} \mathrm{I}$ reactant can be and has been neglected on the relevant energy scale of the experiment. The kinetic energy of the reactants $E_{\mathrm{rel}}$ is given by the average value obtained from imaging the reactant ion and neutral beams. The kinetic energy of the products is calculated from the measured velocity of the $\mathrm{I}^{-}$product, based on conservation of momentum. Since it is proportional to the square of the $\mathrm{I}^{-}$velocity, the $E_{\text {int }}^{\mathrm{CH}_{3} \mathrm{~F}}$-distributions 
average over all scattering angles.

The obtained values are filled with their respective weight $P\left(\left|\vec{v}_{\text {lab }}^{\prime}\right|\right)^{-1} \times\left|\vec{v}_{\text {lab }}^{\prime}-\vec{v}^{\mathrm{cm}}\right| \sin (\theta)$ into one-dimensional histograms to produce the scattering angle and internal energy distributions. The introduction of the Jacobian $\left|\vec{v}_{\text {lab }}^{\prime}-\vec{v}^{\mathrm{cm}}\right| \sin (\theta)$ is a consequence of time slice imaging and accounts for the velocity dependent probability of the scattering events to fall within the detection window. The resolution of the obtained product velocity images and internal energy distributions is dominated by the velocity and angular spreads of the two reactant beams as noted above. Using Gaussian error propagation the accuracy of the $\mathrm{I}^{-}$product velocity is estimated to be between 40 and $60 \mathrm{~m} / \mathrm{s}$ for the range of internal energies and scattering angles under the present conditions. The precise value is different for each scattering event, because it depends not only on the velocity spreads of the two reactant beams, but also on the scattering angle and product velocity magnitude. On this scale simulations show that imperfect velocity mapping and spatial focusing can be safely neglected. Gaussian error propagation also yields an accuracy $\sigma_{\text {int }}$ for the internal energy $E_{\text {int }}$ for each event. To obtain an average accuracy for the whole measured energy distribution we compute the weighted average $\bar{\sigma}_{\text {int }}$ over all events.

The convolution of the true internal energy distribution of the reaction products, which is expected to range from near zero to a maximum internal energy, with the experimental resolution for the imaged velocities leads to a broader measured internal energy distribution that now ranges from zero to a larger maximum internal energy. This also introduces a positive shift of the average product internal energy. Our measurements suggest that in the present case the true internal energy distributions can be roughly approximated by the positive half of a Gaussian distribution. Then the convolution with the (assumed Gaussian) experimental accuracy leads to a shift of the average internal energy of $+\sqrt{2 / \pi} \bar{\sigma}_{\text {int. }}$. This value is used to correct the measured average internal energies for this convolution effect to obtain values that can be compared with simulations. 


\section{Atomistic simulations}

Direct dynamics classical trajectory simulations ${ }^{36}$ were performed for reaction (2) to compare with the experimental results at the representative low and high collision energies of 0.32 and $1.53 \mathrm{eV}$. The simulations were performed at the DFT/B97-1/ECP/d level of electronic structure theory. ${ }^{30}$ This method utilizes the aug-cc-pVDZ basis set ${ }^{45,46}$ for the $\mathrm{C}, \mathrm{H}$, and $\mathrm{F}$ atoms, an effective core potential (ECP) for the core electrons of iodine, ${ }^{47}$ and a $3 \mathrm{~s}, 3 \mathrm{p}$ basis for its valence electrons. The latter was augmented by a d-polarization function with a 0.262 exponent and s, p, and diffuse functions with exponents of $0.034,0.039$, and 0.0873 , respectively. ${ }^{48}$ A range of functionals were compared for use in the DFT direct dynamics simulations and B97-1 ${ }^{49}$ was found to give the best agreement with the high-level $\operatorname{CCSD}(\mathrm{T})^{50}$ energies. $^{30}$

Both the DFT and CSSD(T) calculations indicate that the potential energy surface (PES) for $\mathrm{F}^{-}+\mathrm{CH}_{3} \mathrm{I}$ is different than the traditional $\mathrm{S}_{\mathrm{N}} 2$ PES. ${ }^{51}$ The former does not have $\mathrm{C}_{3 v}$ stationary points for the $\mathrm{F}^{-}-\mathrm{CH}_{3} \mathrm{I}$ complex and $\left[\mathrm{F}-\mathrm{CH}_{3}-\mathrm{I}\right]^{-}$central barrier. There is a pre-reaction complex, but it has a hydrogen-bonded $\mathrm{F}^{-}-\mathrm{HCH}_{2} \mathrm{I} \mathrm{C}$ structure. This potential minimum is connected to the traditional $\mathrm{C}_{3 v}$ post-reaction $\mathrm{FCH}_{3}-\mathrm{I}^{-}$ion-dipole complex via a $\mathrm{C}_{s}\left[\mathrm{~F}-\mathrm{HCH}_{2}-\mathrm{I}\right]^{-}$transition state. ${ }^{30}$ The $0 \mathrm{~K}$ DFT/B97-1/ECP/d relative energies, without zero-point energies, for $\mathrm{F}^{-}+$ $\mathrm{CH}_{3} \mathrm{I}, \mathrm{F}^{-}-\mathrm{HCH}_{2} \mathrm{I},\left[\mathrm{F}-\mathrm{HCH}_{2}-\mathrm{I}\right]^{-}, \mathrm{FCH}_{3}-\mathrm{I}^{-}$, and $\mathrm{FCH}_{3}+\mathrm{I}^{-}$are 0.0, -84.5, -74.5, -226.4, and $-195.4 \mathrm{~kJ} / \mathrm{mol}$.

For a direct comparison with the experiments at the collision energies of 0.32 and $1.53 \mathrm{eV}$, the simulations were performed for $\mathrm{CH}_{3} \mathrm{I}$ with a vibrational temperature of $360 \mathrm{~K}$ and a rotational temperature of $75 \mathrm{~K}$. Quasiclassical sampling ${ }^{52}$ was used to determine initial conditions for the trajectories, as described previously for the $\mathrm{Cl}^{-}+\mathrm{CH}_{3} \mathrm{I}$ simulation ${ }^{10}$ and the preliminary report of the $\mathrm{F}^{-}+\mathrm{CH}_{3} \mathrm{I}$ simulation. ${ }^{11}$ The simulations were performed using the VENUS general chemical dynamics computer program ${ }^{53,54}$ interfaced to the NWChem electronic structure computer

program. ${ }^{55,56}$ Reactive trajectories were identified by animating the trajectories and determining their atomic-level motions. A total of 1250 and 1600 trajectories were calculated for the 0.32 and $1.53 \mathrm{eV}$ collision energies, respectively. 
Instead of sampling the impact parameter $b$ randomly, the trajectories were calculated at fixed $b$ of $1,2,3,4,5,7,8,8.5$, and $8.75 \AA$ for $E_{\text {rel }}=0.32 \mathrm{eV}$ and $1,2,3,4,5,5.5,5.75$, and $6 \AA$ for $E_{\text {rel }}=1.53 \mathrm{eV}$. There were no reactions out of 200 trajectories at $b=8.75 \AA$ for the lower $E_{\text {rel }}$ and at $b=6 \AA$ for the higher $E_{\text {rel. }}$. It is of interest that these values for $b_{\max }$ are similar to those of 9.0 and $6.0 \AA$ found by assuming that the $\mathrm{F}^{-}+\mathrm{CH}_{3} \mathrm{I}$ orbital angular momentum is adding to the two large moment of inertia rotational degrees of freedom, which gives rise to a centrifugal potential at the $\left[\mathrm{F}-\mathrm{HCH}_{2}-\mathrm{I}\right]^{-}$transition state.

\section{Results}

\section{Ion Imaging Experiments}

By integrating over several times $10^{5}$ scattering events of reaction (2) per collision energy, we obtain the velocity images of the $\mathrm{I}^{-}$reaction product, as described above. These data are depicted in Fig. 2 for collision energies between 0.32 and $2.34 \mathrm{eV}$. The color code indicates the number of events per histogram bin. The product ion distributions are presented in the center-of-mass frame, where the relative velocity vector is aligned along the x-axis of the histogram (see the Newton diagram in Fig. 2). The dashed concentric rings in Fig. 2, A1-E1 mark spheres of same product excitation. They are spaced at $1.0 \mathrm{eV}$ intervals. The outermost ring marks the kinematical cutoff, i. e. the largest possible product velocity based on energy conservation.

For the lowest studied relative collision energies of $E_{\text {rel }}=0.32 \mathrm{eV}$ and $E_{\text {rel }}=0.69 \mathrm{eV}$ much smaller $\mathrm{I}^{-}$product velocities than corresponding to the kinematical cutoff dominate the differential cross section, as seen from the images in Fig. 2, A1\&B1. For the three higher relative collision energies between $1.53 \mathrm{eV}$ and $2.34 \mathrm{eV}$ we find a broad distribution of product velocities reaching from standstill all the way to the kinematical cutoff (Fig. 2, C1-E1). The distribution is smooth and decreases in amplitude for increasing product velocities. The reactions are observed to occur into all scattering angles. For relative energies of $0.69 \mathrm{eV}$ and above backward scattering into a wide range of angles becomes increasingly dominant. This is derived from the normalized scattering 
angle histograms depicted in Fig. 2, A2-E2.

The total amount of energy is not sufficient to allow for dissociation of the molecular product $\mathrm{CH}_{3} \mathrm{~F}$ in the $\mathrm{I}^{-}$product channel of reaction (2) for all except the highest collision energy in this study. Hence the energy missing in the translational motion of the reaction products is distributed to the $\mathrm{CH}_{3} \mathrm{~F}$ rotational and vibrational degrees of freedom. For relative collision energies $E_{\text {rel }}>$ $1.8 \mathrm{eV}$ the product channel $\mathrm{F}^{-}+\mathrm{CH}_{3} \mathrm{I} \longrightarrow \mathrm{I}^{-}+\mathrm{CH}_{2}+\mathrm{HF}$ is energetically accessible. Its kinematic cutoff is indicated by the solid circle in Fig. 2, E1, which shows the differential cross section for $2.34 \mathrm{eV}$ relative collision energy. As seen from that image, we observe a significant amount of scattering events within this cutoff. Since no qualitative change is noticed in the differential cross section as compared to the lower collision energy of $E_{\text {rel }}=1.81 \mathrm{eV}$, we interpret this as the appearance of transient $\mathrm{CH}_{3} \mathrm{~F}$ products, which subsequently decay into $\mathrm{CH}_{2}+\mathrm{HF}$.

To quantify the redistribution of energy into product translation and internal excitation, we derive the distributions of the internal energy of the $\mathrm{CH}_{3} \mathrm{~F}$ products. The normalized distributions, which contain all scattering events for one relative collision energy, are shown in Fig. 2, A3-E3. Vanishing internal product excitation $E_{\text {int }}^{\mathrm{CH}_{3} \mathrm{~F}}=0$ occurs at the kinematical cutoff in Fig. 2, A1E1, where the kinetic energy stored in relative product translation is maximal. On the other hand maximal internal product excitation $E_{\text {int }}^{\mathrm{CH}_{3} \mathrm{~F}}=E_{\text {rel }}-\Delta H$ occurs if the reaction products are formed at standstill in the center-of-mass frame. This corresponds to events in the center of the images in Fig. 2, A1-E1 and accounts for the sharp cutoff in the histograms Fig. 2, A3-E3. The measured $E_{\text {int }}^{\mathrm{CH}_{3} \mathrm{~F}}$-distributions are bound by these two limits except for broadening due to the finite energy resolution.

The $E_{\text {int }}^{\mathrm{CH}_{3} \mathrm{~F}}$-distributions shown as right column of Fig. 2 in panels A3 through E3 (black lines) reveal that for the two lowest collision energies of 0.32 and $0.69 \mathrm{eV}$ most scattering events feature very high internal excitation of the $\mathrm{CH}_{3} \mathrm{~F}$ product close to the maximal possible value. For the three higher collision energies $1.53 \mathrm{eV}, 1.81 \mathrm{eV}$ and $2.34 \mathrm{eV}$ we observe a broad distribution of internal excitations. On average, however, also in these cases energy is preferentially distributed to internal rather than translational degrees of freedom. It should be noted that the one-dimensional 
$E_{\text {int }}^{\mathrm{CH}_{3} \mathrm{~F}}$ distributions do not peak at maximal energy transfer, while the two-dimensional images in the left column of Fig. 2 peak at zero velocity. This is a consequence of the Jacobian in the integral over the angular coordinates (see section on data analysis).

Table 1 lists the average values of the experimental $E_{\text {int }}$-distributions in Fig. 2, A3-E3. These values are corrected for the finite energy resolution in the experiment, as described in the data analysis section. The results are also shown in Table 1. The corrected energies are about $10 \%$ higher (right column in Table 1). The measured average internal product excitation $\bar{E}_{\text {int }}$ increases with collision energy from $1.52 \mathrm{eV}$ at $E_{\mathrm{rel}}=0.32 \mathrm{eV}$ to $2.58 \mathrm{eV}$ at $E_{\mathrm{rel}}=2.34 \mathrm{eV}$. For the two lowest collision energies of $0.32 \mathrm{eV}$ and $0.69 \mathrm{eV}$, on average about $70 \%$ of the total available energy is transformed into internal product excitation. For the higher collision energies between 1.53 and $2.34 \mathrm{eV}$, where increasingly higher absolute internal energies of the $\mathrm{CH}_{3} \mathrm{~F}$ reaction product are observed, the fraction decreases slightly to about $60 \%$ of the total available energy.

\section{Direct Chemical Dynamics Simulations}

\section{Reaction probabilities and cross sections}

The classical trajectory simulations reveal for both the 0.32 and $1.53 \mathrm{eV}$ collision energies three dominant atomic-level reaction mechanisms: direct stripping, direct rebound, and an indirect pathway in which formation of the $\mathrm{F}^{-}-\mathrm{HCH}_{2} \mathrm{I}$ complex is important. $\mathrm{F}^{-}$strikes the backside of $\mathrm{CH}_{3} \mathrm{I}$ and directly displaces $\mathrm{I}^{-}$for the rebound mechanism. For stripping $\mathrm{F}^{-}$approaches $\mathrm{CH}_{3} \mathrm{I}$ from the side and directly strips away the $\mathrm{CH}_{3}$ group. The probabilities of these three mechanisms versus impact parameter, $P_{r}(b)$, are plotted in the top row of Fig. 3 for 0.32 and $1.53 \mathrm{eV}$ collision energy. The rebound mechanism occurs at small impact parameters, while the stripping mechanism becomes important for large impact parameters. The indirect mechanism occurs for all impact parameters up to $b_{\max }$. The direct rebound mechanism has been observed in previous simulations of the $\mathrm{Cl}^{-}+\mathrm{CH}_{3} \mathrm{Y}(\mathrm{Y}=\mathrm{Cl}, \mathrm{Br}, \mathrm{I}) \mathrm{S}_{\mathrm{N}} 2$ reactions. ${ }^{10,57-59}$ The stripping mechanism was seen for $\mathrm{Cl}^{-}$ $+\mathrm{CH}_{3} \mathrm{Cl}$ at high collision energies. ${ }^{59}$

Though the vast majority of the indirect reaction occurred by solely forming the $\mathrm{F}^{-}-\mathrm{HCH}_{2} \mathrm{I}$ 
hydrogen-bonded complex, there were also other pathways. They involve formation of the $\mathrm{FCH}_{3}-$ $\mathrm{I}^{-}$post-reaction complex, recrossing of the $\left[\mathrm{F}-\mathrm{HCH}_{2}-\mathrm{I}\right]^{-}$barrier, the roundabout mechanism, ${ }^{10}$ and a half-roundabout mechanism in which $\mathrm{F}^{-}$strikes the I-atom of $\mathrm{CH}_{3} \mathrm{I}$ and then reacts with the backside of $\mathrm{CH}_{3} \mathrm{I}$ after $\mathrm{CH}_{3}$ completes a one-half rotation about the I-atom. Some of the indirect pathways were composites of these mechanisms. Identifying the $\mathrm{F}^{-}-\mathrm{HCH}_{2} \mathrm{I}$ complex as A, the $\mathrm{FCH}_{3}-\mathrm{I}^{-}$complex as $\mathrm{B}$, roundabout as RA, half-roundabout as h-RA, and barrier recrossing as $\mathrm{br}$, the following are the fractions of reaction by the different indirect pathways at $E_{\mathrm{rel}}=0.32 \mathrm{eV}$ : A, 0.537; B, 0.006; A+B, 0.017; A+B+br, 0.017, h-RA, 0.006; h-RA+A, 0.004; h-RA+B,0.001; and h-RA+A+B, 0.001. The total indirect fraction is 0.589 , of which only forming $\mathrm{F}^{-}-\mathrm{HCH}_{2} \mathrm{I}$ comprises $91 \%$. For the higher $1.53 \mathrm{eV}$ collision energy the fractions of the different types of indirect events are: A, 0.591; A+B, 0.006; RA, 0.003; h-RA, 0.027, RA+A, 0.006; and RA+B, 0.003. Here the total indirect fraction is 0.636 , for which only forming $\mathrm{F}^{-}-\mathrm{HCH}_{2} \mathrm{I}$ comprises $92 \%$, similar as for the $0.32 \mathrm{eV}$ simulations.

The reaction cross section $\sigma_{r}$ was obtained by integrating the total $P_{r}(b)$ over the impact parameter according to $\int P_{r}(b) 2 \pi b d b$, and the resulting value of $\sigma_{r}$ is $108.7 \pm 9.7$ and $8.6 \pm 2.2 \AA^{2}$ at $E_{\text {rel }}$ of 0.32 and $1.53 \mathrm{eV}$, respectively. There is more than an order of magnitude decrease in the reactive cross section upon increasing $E_{\text {rel }}$ from 0.32 to $1.53 \mathrm{eV}$. The cross sections for the individual direct rebound, direct stripping, and indirect pathways are, respectively, $16.0 \pm 2.3,27.5 \pm 4.3$, and 65.2 $\pm 6.5 \AA^{2}$ at $E_{\text {rel }}=0.32 \mathrm{eV}$ and $2.5 \pm 0.7,1.0 \pm 0.3$, and $5.1 \pm 1.7 \AA^{2}$ at $E_{\text {rel }}=1.53 \mathrm{eV}$. The cross

sections reported here for $E_{\text {rel }}=0.32 \mathrm{eV}$ are slightly different than those reported previously, ${ }^{11}$ as a result of a more accurate integration of $P_{r}(b)$ over the impact parameter. The largest difference is for the indirect pathway cross section, which is $7 \%$ larger.

\section{Product energy partitioning}

The simulation results for the product energy partitioning are shown in the center row of Fig. 3 and are summarized in Table 2. The total distributions are also overlaid in Fig. 2, A3 and C3 (blue lines). We find that the overall fraction partitioned to internal excitation of $\mathrm{CH}_{3} \mathrm{~F}$ is $0.67-0.71$ and 
$0.58-0.66$ for 0.32 and $1.53 \mathrm{eV}$ collision energy, respectively. For $E_{\text {rel }}=0.32 \mathrm{eV}$ this partitioning is primarily into vibration with a fraction of $0.62 \pm 0.02$. The fraction of rotational excitation is $0.07 \pm 0.01$. Partitioning of energy to rotation becomes more important for $E_{\text {rel }}=1.53 \mathrm{eV}$, with fractions of $0.49 \pm 0.06$ and $0.13 \pm 0.03$ for vibration and rotation, respectively.

The simulations show that the direct rebound, direct stripping, and indirect atomic-level mechanisms have different product energy partitioning, as shown in Table 2. At $E_{\text {rel }}=0.32 \mathrm{eV}$ the energy partitioning for direct rebound and direct stripping are similar, with a small fraction partitioned to $\mathrm{CH}_{3} \mathrm{~F}$ rotation and similar partitioning to $\mathrm{CH}_{3} \mathrm{~F}$ vibration and relative translation. For the indirect mechanism the transfer to $\mathrm{CH}_{3} \mathrm{~F}$ rotation remains low, but the transfer to $\mathrm{CH}_{3} \mathrm{~F}$ vibration and relative translation are increased and decreased, respectively.

There are substantial differences between the energy partitioning at $E_{\text {rel }}=1.53 \mathrm{eV}$ and $0.32 \mathrm{eV}$. At the higher $E_{\text {rel }}$ energy partitioning to rotation increases for the direct rebound and direct stripping mechanisms, but remains the same for the indirect mechanism. Interestingly, at $E_{\text {rel }}=1.53 \mathrm{eV}$ the partitioning of energy to $\mathrm{CH}_{3} \mathrm{~F}$ vibration and relative translation is different for the direct rebound and direct stripping mechanisms. Transfer to relative translation is more important for the direct rebound mechanism, while transfer to $\mathrm{CH}_{3} \mathrm{~F}$ vibration is more important for the direct stripping mechanism. For the indirect mechanism a smaller fraction of energy is transferred to $\mathrm{CH}_{3} \mathrm{~F}$ vibration at $E_{\text {rel }}$ of $1.53 \mathrm{eV}$ as compared to $0.32 \mathrm{eV}$.

\section{Velocity scattering angle distribution}

The simulated velocity scattering angle distributions versus $\cos \theta$ are given in the bottom row of Fig. 3 for both $E_{\text {rel }}$ of 0.32 and $1.53 \mathrm{eV}$. Distributions are given for the total scattering and for the scattering of the three atomic-level mechanisms. The total distributions are also overlaid in Fig. 2, A2 and C2 (blue lines).

For both collision energies the scattering for the direct rebound mechanism is backward with $\cos \theta$ between 0.0 and -1.0 . The scattering for the direct stripping mechanism is in the forward direction with $\cos \theta$ in the range of 0.0 to 1.0 , except for one trajectory with $\cos \theta=-0.1$. As 
described previously, ${ }^{11}$ for this trajectory there is a strong attractive interaction as $\mathrm{F}^{-}$approaches the side of $\mathrm{CH}_{3} \mathrm{I}$, changing the relative velocity vector and resulting in backward scattering with $\theta=98^{\circ}$. Thus, a small fraction of the stripping may be slightly scattered in the backward direction.

The indirect scattering covers the complete range of $\cos \theta$. For $E_{\mathrm{rel}}=0.32 \mathrm{eV}$ the scattering is isotropic and most probable values are at the 1.0 and -1.0 limiting values of $\cos \theta$. The indirect scattering is not isotropic at $E_{\mathrm{rel}}$ of $1.53 \mathrm{eV}$, with forward scattering being more probable, while the total scattering is isotropic at both collision energies.

\section{Discussion}

\section{Comparison between experiment and simulation}

The detailed results of the presented scattering experiments and trajectory simulations allow for an unprecedented comparison of the product energy and angular distributions. When comparing Tables 1 and 2 we find that the average fraction of energy that is partitioned to internal energy amounts to $70 \pm 9 \%(59 \pm 8 \%)$ in the experiment and $69 \pm 2 \%(63 \pm 4 \%)$ in the simulations for $0.32(1.53 \mathrm{eV})$ collision energy. This shows a very good quantitative agreement between experiment and theory within their respective accuracies. Note that the correction due to the finite energy resolution of the experiment does not induce a large shift in the average internal energies.

The simulations show that between $80-90 \%$ of the internal energy released to the $\mathrm{CH}_{3} \mathrm{~F}$ product is transferred to its vibrational degrees of freedom. For the three highest collision energies studied in the experiment, the average internal product excitation exceeds the energy gained from the reaction exothermicity $\bar{E}_{\text {int }}>-\Delta H$. This shows that during the reaction, energy is efficiently transferred from reactant translation to internal vibrational excitation of the product.

For $0.32 \mathrm{eV}$ relative collision energy, not only the average internal energies but also the probability distribution of the internal energy agrees well between experiment and simulation (see Fig. 2, A3). The experimental distribution extends to smaller values of the internal energy, which corresponds to larger velocities in translation. This is a consequence of the broadening of the measured 
velocity distribution due to the finite energy width of the reactant beams (see section on experimental procedure). The simulated distribution has not been convoluted with the experimental accuracy. For the $1.53 \mathrm{eV}$ collision energy the experiment finds significantly more probability for low internal energies than the simulation (see Fig. 2, C3). This can not be explained by the energy resolution. It corresponds to measured events that are backward-scattered with large product velocities in the two-dimensional velocity distribution and that are also observed in the distribution of the scattering angle.

The experimental and simulated distributions of the scattering angle (see Fig. 2, A2 and C2) show roughly a similar shape. In both cases scattering occurs in all directions with an almost isotropic distribution for the simulations and a backward-dominated distribution in the measurements. The simulations show that the rather isotropic scattering is not only caused by the indirect mechanism, but by a sum of stripping, rebound, and indirect scattering. In more detail, one finds that for $E_{\text {rel }}=0.32 \mathrm{eV}$ the scattering is most probable for $\cos \theta$ close to 1.0 in the simulations, but for values near -1.0 in the experiments. The stronger backward-scattering in the experiments also extends over a larger scattering angle interval. The difference between the measured and simulated scattering angle distributions is more pronounced at $E_{\mathrm{rel}}=1.53 \mathrm{eV}$. There the scattering is again quite isotropic for the simulations caused by the sum of the forward-scattered stripping, the backward-scattered rebound reactions and the large contribution of indirect scattering. In the experiment one finds strong backward scattering and some forward scattering, which can be attributed to a combination of the indirect mechanism and the direct rebound mechanism with roughly similar probability. The observed differences in the angular distributions and in the internal energy distribution for $1.53 \mathrm{eV}$ could therefore be explained by a difference in the branching ratios for the forward-scattered direct stripping mechanism and the backward-scattered direct rebound mechanism in the experiment compared to the simulation. Further chemical dynamics simulations and crossed-beam scattering experiments at higher resolution may clarify this in the future. In particular, we will investigate the unimolecular dynamics of the $\mathrm{F}^{-}-\mathrm{HCH}_{2} \mathrm{I}$ hydrogen-bonded intermediate in more detail and consider additional quantum chemical theories for the $\mathrm{F}^{-}+\mathrm{CH}_{3} \mathrm{I}$ 
direct dynamics.

\section{Comparison with other $S_{N} 2$ Reactions}

It is interesting to compare the reaction of $\mathrm{F}^{-}$with $\mathrm{CH}_{3} \mathrm{I}$ studied here with the related $\mathrm{S}_{\mathrm{N}} 2$ reactions of $\mathrm{Cl}^{-}+\mathrm{CH}_{3} \mathrm{I}$ (central barrier: $\left.+0.31 \mathrm{eV}\right), \mathrm{F}^{-}+\mathrm{CH}_{3} \mathrm{Cl}$ (central barrier: $+0.1 \mathrm{eV}^{60}$ ), and $\mathrm{Cl}^{-}$ $+\mathrm{CH}_{3} \mathrm{Br}$ (central barrier: $+0.40 \mathrm{eV}^{61}$ ). The first has been recently studied with crossed-beam slice imaging and compared to direct dynamics simulations. ${ }^{10}$ The second has been the subject of detailed trajectory simulations ${ }^{62}$ and a guided ion beam study, ${ }^{29}$ and the third has been extensively studied by ion beam mass spectrometry ${ }^{20,24}$ and multiple trajectory simulations. ${ }^{59,63}$

For low collision energies $E_{\text {rel }}<0.5 \mathrm{eV}$, these other systems show formation of an entrance channel complex, which is much longer lived than its rotational period. This results in isotropic scattering and a high internal and low translational excitation of the molecular product, in agreement with the findings of this study. For $\mathrm{Cl}^{-}+\mathrm{CH}_{3} \mathrm{I}$ the experimentally determined partitioning into translational and internal energy even agrees with the prediction from a phase space theory (PST) calculation. ${ }^{10}$ For higher collision energies $(0.70 \mathrm{eV})$ the calculation of $\mathrm{F}^{-}+\mathrm{CH}_{3} \mathrm{Cl}$ yields shorter complex lifetimes and backward scattering of the anionic product. The increased relative kinetic energy of the reactants also results in larger product translational energy. This corresponds to the experimental findings for $\mathrm{Cl}^{-}+\mathrm{CH}_{3} \mathrm{I}$ for 0.8 to $1.9 \mathrm{eV}$ relative energy, which show a dominating direct reaction mechanism with little coupling to the internal quantum states of the molecular product. ${ }^{10}$

For the reaction $\mathrm{F}^{-}+\mathrm{CH}_{3} \mathrm{Cl} \rightarrow \mathrm{CH}_{3} \mathrm{~F}+\mathrm{Cl}^{-}$, which is also a highly exothermic $\mathrm{S}_{\mathrm{N}} 2$ reaction with a small central barrier, a large cross section was found in guided ion beam experiments at low relative collision energies. ${ }^{29}$ Using quantum chemistry calculations of stationary points the authors recognized that hydrogen-bonded complexes will deepen the entrance well and suggested that this may help to stabilize prereactive $\mathrm{S}_{\mathrm{N}} 2$ intermediates at the lowest collision energies. For collision energies above $0.4 \mathrm{eV}$ they reported a rapid decrease of the reaction cross section consistent with the transition from a complex-mediated process to a direct backside $\mathrm{S}_{\mathrm{N}} 2$ mechanism, which im- 
poses stricter stereodynamic constraints. This is in line with earlier studies using kinetic energy release Fourier transform ion cyclotron resonance spectroscopy ${ }^{22}$ and ab initio direct dynamics calculations ${ }^{64}$ that reported high translational and low internal excitation of the reaction products $\mathrm{Cl}^{-}$and $\mathrm{CH}_{3} \mathrm{~F}$. These findings are, however, in contrast to the observations in our crossed-beam experiments.

The dependence of the $\mathrm{F}^{-}+\mathrm{CH}_{3} \mathrm{I} \rightarrow \mathrm{CH}_{3} \mathrm{~F}+\mathrm{I}^{-}$product energy partitioning on the collision energy also differs significantly with findings for $\mathrm{Cl}^{-}+\mathrm{CH}_{3} \mathrm{Br} \mathrm{S}_{\mathrm{N}} 2$ substitution. ${ }^{20,24,59,63}$ For low collision energies and low excitation energies of the $\mathrm{Cl}^{-}-\mathrm{CH}_{3} \mathrm{Br}$ pre-reaction complex the available energy is preferentially partitioned to $\mathrm{CH}_{3} \mathrm{Cl}$ vibration with a fraction higher than the prediction of PST. However, as the collision energy is increased the partitioning to $\mathrm{CH}_{3} \mathrm{Cl}$ vibration dramatically decreases; e.g. from the simulations ${ }^{59}$ the fraction partitioned to $\mathrm{CH}_{3} \mathrm{Cl}$ vibration is 0.80 and 0.26 for the respective collision energies of 0.04 and $2.17 \mathrm{eV}$. In sharp contrast, for the $\mathrm{F}^{-}$ $+\mathrm{CH}_{3} \mathrm{I}$ experiments the partitioning to $\mathrm{CH}_{3} \mathrm{~F}$ internal energy only slightly decreases from 0.70 to 0.62 as the collision energy is increased from 0.32 to $2.34 \mathrm{eV}$.

For the lowest collision energy in the present study the nearly isotropic scattering and high internal excitation of the molecular reaction product seems to be in qualitative agreement with the experimental observation for $\mathrm{Cl}^{-}+\mathrm{CH}_{3}$ I. However, the trajectory simulations show that in fact three different atomic-level mechanisms are involved in the dynamics. For the higher collision energies the observed dynamics for reaction (2) deviate strongly from the $\mathrm{Cl}^{-}+\mathrm{CH}_{3} \mathrm{I}_{\text {and }} \mathrm{F}^{-}+$ $\mathrm{CH}_{3} \mathrm{Cl}$ reactions, both with respect to the scattering angle distribution and the product translational energy. Instead of finding a dominating backward-scattered direct rebound mechanism, the indirect mechanism is similarly important for reaction (2) as the rebound mechanism, and also the direct stripping mechanism plays a role.

Our finding of the importance of an $\mathrm{H}$-bond assisted mechanism in the $\mathrm{F}^{-}+\mathrm{CH}_{3} \mathrm{I} \mathrm{S}_{\mathrm{N}} 2$ reaction might be reminiscent of a current discussion in the field of reactive scattering of neutrals. In a recent crossed beam study it was quite surprisingly found that vibrational excitation of the $\mathrm{CH}$ stretch in the $\mathrm{F}+\mathrm{CHD}_{3}$ reaction suppressed the $\mathrm{HF}+\mathrm{CD}_{3}$ product channel. ${ }^{65}$ This is in stark contrast to the 
expectation from conventional transition state theory, which would predict an enhancement of $\mathrm{H}$ abstraction. The entrance channel van der Waals well, corresponding to an H-bonded intermediate, plays a significant role in the dynamics at the low collision energy in the study. Using classical trajectory calculations it could be shown that a stereodynamic effect associated with the H-bonded intermediate steers the trajectories away from the excited $\mathrm{CH}$ bond, thereby suppressing hydrogen abstraction. 66

In the present case, the configuration of the H-bonded complex is quite different from the transition state. However, the Born-Oppenheimer potential energy hyper-surface is found to be very flat in this region. ${ }^{30}$ Chemical intuition suggests that the transition from the H-bonded complex to the transition state involves the extremely floppy $\mathrm{A}^{\prime} \mathrm{F}^{-}$bend vibration, which is the least energetic vibrational mode of the complex at only $80 \mathrm{~cm}^{-1}$ (see Table 3 in Ref. ${ }^{30}$ ). The classical $\mathrm{S}_{\mathrm{N}} 2$ mechanism, i.e. the direct rebound, occurs for impact parameters up to $5.0 \AA$ ( $4 \AA$ ) for the low (high) collision energy (see upper panels in Fig. 3). On the other hand the H-bond assisted indirect mechanism occurs over a broader range of impact parameters up to $8.5 \AA$ and $5 \AA$ for the two collision energies (see Fig. 3). This suggests that the transient $\mathrm{H}$-bond in fact assists in funneling the $\mathrm{F}^{-}$ anion into the $\mathrm{CH}_{3}$ umbrella, i.e. towards the transition state where inversion occurs (see typical trajectory in Fig. 4 of Ref. ${ }^{11}$ ). This notion is supported by the large cross section of $108.7 \pm 9.7 \AA^{2}$, which has in fact reached the respective capture limit of $105.0 \AA^{2} .67$

Given the large exothermicity of the $\mathrm{F}^{-}+\mathrm{CH}_{3} \mathrm{I}$ reaction and the shallow $\mathrm{F}^{-}-\mathrm{HCH}_{2} \mathrm{I}$ potential minimum of only $0.1 \mathrm{eV}$, it is quite surprising that a large fraction of the reaction occurs indirectly at the high collision energy of $1.53 \mathrm{eV}$ and above. This is suggestive of non-statistical dynamics for the $\mathrm{F}^{-}-\mathrm{HCH}_{2} \mathrm{I}$ pre-reaction complex, as found in previous studies for the $\mathrm{Cl}^{-}-\mathrm{CH}_{3} \mathrm{Cl}^{68}$ and $\mathrm{Cl}^{-}-\mathrm{CH}_{3} \mathrm{Br}^{63}$ pre-reaction complexes. Because of the disparity between the low frequency intermolecular modes and the higher frequency intramolecular modes of these complexes, there exists a bottleneck ${ }^{5}$ for attaining the $\mathrm{S}_{\mathrm{N}} 2$ transition state. The $\mathrm{F}^{-}-\mathrm{HCH}_{2} \mathrm{I}$ complex has a deep $1 \mathrm{eV}$ minimum with respect to the $\mathrm{F}^{-}+\mathrm{CH}_{3} \mathrm{I}$ reactants, but a shallow $0.1 \mathrm{eV}$ minimum with respect to the $\left[\mathrm{F}-\mathrm{HCH}_{2}-\mathrm{I}\right]^{-}$transition state. However, if energy transfer from the intermolecular modes of the 
$\mathrm{F}^{-}-\mathrm{HCH}_{2} \mathrm{I}$ complex to the $\mathrm{C}-\mathrm{I}$ stretch is inefficient, the lifetime of the $\mathrm{F}^{-}-\mathrm{HCH}_{2} \mathrm{I}$ complex may be much longer than the prediction of statistical theory. The indirect fraction of the $\mathrm{F}^{-}+\mathrm{CH}_{3} \mathrm{I}$ reaction is $60 \%$ at both the 0.32 and $1.53 \mathrm{eV}$ collision energies, which is consistent with such nonstatistical dynamics. In future work it will be of interest to calculate the anharmonic RRKM rate constant for $\mathrm{F}^{-}-\mathrm{HCH}_{2} \mathrm{I} \rightarrow \mathrm{CH}_{3} \mathrm{~F}+\mathrm{I}^{-}$dissociation versus energy and to investigate the coupling between the $\mathrm{F}^{-}-\mathrm{HCH}_{2} \mathrm{I}$ intermolecular modes and the $\mathrm{C}-\mathrm{I}$ stretch.

\section{Conclusion}

The differential scattering cross section for the highly exoergic nucleophilic substitution reaction of fluorine anions with methyl iodide forming iodine anions has been measured as a function of the collision energy using crossed-beam velocity map ion imaging. High level direct dynamics classical trajectory simulations have been performed and compared with experiment. We find that reactive scattering occurs for about half of the products by indirect dynamics even at high relative collision energies. The classical direct, co-linear reaction mechanism that visualizes $\mathrm{S}_{\mathrm{N}}$ 2-reactions in organic chemistry text books forms most of the remaining products, while a third mechanism that occurs by direct stripping also plays a role. The evidence for this stems from the observed agreement of the internal energy and scattering angle distributions in experiment and simulation.

The unexpected finding of the strong contribution of indirect reaction dynamics is in contrast to previous studies of related halogen anion-methyl halide $\mathrm{S}_{\mathrm{N}} 2$-reactions and shows that the relative importance of different atomic-level mechanisms may depend strongly on the details of the interaction potential. The simulations further show that the roundabout mechanism ${ }^{10}$ contributes to the total scattering with a probability of a few percent. Indirect reaction dynamics are thus found to be much more important for nucleophilic substitution reactions than previously anticipated.

\section{Acknowledgement}

This work has been supported by the Deutsche Forschungsgemeinschaft under contract No. WE 
2592/3-2. R.O. acknowledges support by the Landesgraduiertenförderung Baden-Württemberg. We thank the University of Freiburg, where the measurements presented here have been carried out, for supporting this research. The direct chemical dynamics simulations reported here are based upon work supported by the National Science Foundation under Grants No. CHE-0615321 and CHE-0957521, and the Robert A. Welch Foundation under Grant No. D-0005. Support was also provided by the High-Performance Computing Center (HPCC) at Texas Tech University, un-

der the direction of Philip W. Smith and the Texas Advanced Computing Center (TACC) at the University of Texas at Austin. Part of this research was performed at the Environmental Molecular Sciences Laboratory (EMSL), a national scientific user facility sponsored by the U.S. Department of Energy's Office of Biological and Environmental Research and located at the Pacific Northwest National Laboratory, operated for the Department of Energy by Battelle.

\section{References}

(1) Vollhardt, K. P. C.; Schore, N. E. Organic Chemistry, Structure and Function; Pallgrave Macmillan, 2003.

(2) Chabinyc, M. L.; Craig, S. L.; Regan, C. K.; Brauman, J. I. Science 1998, 279, 1882-1886.

(3) Laerdahl, J. K.; Uggerud, E. Int. J. Mass Spectrom. 2002, 214, 277-314.

(4) Nettey, S.; Swift, C. A.; Joviliano, R.; Noin, D. O.; Gronert, S. J. Am. Chem. Soc. 2012, 134, 9303-9310.

(5) Hase, W. L. Science 1994, 266, 998-1002.

(6) Schmatz, S. Chem. Phys. Chem. 2004, 5, 600-617.

(7) Manikandan, P.; Zhang, J.; Hase, W. L. J. Phys. Chem. A 2012, 116, 3061-3080.

(8) Mikosch, J.; Frühling, U.; Trippel, S.; Schwalm, D.; Weidemüller, M.; Wester, R. Phys. Chem. Chem. Phys. 2006, 8, 2990-2999. 
(9) Pei, L.; Farrar, J. M. J. Chem. Phys. 2012, 136, 204305.

(10) Mikosch, J.; Trippel, S.; Eichhorn, C.; Otto, R.; Lourderaj, U.; Zhang, J. X.; Hase, W. L.; Weidemüller, M.; Wester, R. Science 2008, 319, 183-186.

(11) Zhang, J.; Mikosch, J.; Trippel, S.; Otto, R.; Weidemueller, M.; Wester, R.; Hase, W. L. J. Phys. Chem. Lett. 2010, 1, 2747-2752.

(12) Otto, R.; Brox, J.; Stei, M.; Trippel, S.; Best, T.; Wester, R. Nature Chemistry 2012, 4, 534538.

(13) Mikosch, J.; Weidemüller, M.; Wester, R. Int. Rev. Phys. Chem. 2010, 29, 589-617.

(14) Olmstead, W. N.; Brauman, J. I. J. Am. Chem. Soc. 1977, 99, 4219-4228.

(15) DePuy, C. H.; Gronert, S.; Mullin, A.; Bierbaum, V. M. J. Am. Chem. Soc. 1990, 112, 86508655 .

(16) Morris, R. A.; Viggiano, A. A. J. Phys. Chem. 1994, 98, 3740-3746.

(17) Kato, S.; Davico, G. E.; Lee, H. S.; DePuy, C. H.; Bierbaum, V. M. Int. J. Mass Spectrom. 2001, 210/211, 223-229.

(18) Barlow, S. E.; Doren, J. M. V.; Bierbaum, V. M. J. Am. Chem. Soc. 1988, 110, 7240-7242.

(19) Viggiano, A. A.; Morris, R. A.; Paschkewitz, J. S.; Paulson, J. F. J. Am. Chem. Soc. 1992, $114,10477-10482$.

(20) Graul, S. T.; Bowers, M. T. J. Am. Chem. Soc. 1994, 116, 3875-3883.

(21) Craig, S. L.; Brauman, J. I. Science 1997, 276, 1536-1538.

(22) Vanorden, S. L.; Pope, R. M.; Buckner, S. W. J. Mass Spectrom. 1991, 26, 1003-1007.

(23) Tonner, D. S.; McMahon, T. B. J. Am. Chem. Soc. 2000, 122, 8783-8784. 
(24) Angel, L. A.; Ervin, K. M. J. Am. Chem. Soc. 2003, 125, 1014-1027.

(25) Wester, R.; Bragg, A. E.; Davis, A. V.; Neumark, D. M. J. Chem. Phys. 2003, 119, 1003210039.

(26) Le Garrec, J. L.; Rowe, B. R.; Queffelec, J. L.; Mitchell, J. B. A.; Clary, D. C. J. Chem. Phys. 1997, 107, 1021-1024.

(27) Otto, R.; Mikosch, J.; Trippel, S.; Weidemüller, M.; Wester, R. Phys. Rev. Lett. 2008, 101, 063201.

(28) Mikosch, J.; Otto, R.; Trippel, S.; Eichhorn, C.; Weidemüller, M.; Wester, R. J. Phys. Chem. A 2008, 112, 10448-10452.

(29) Angel, L. A.; Ervin, K. M. J. Phys. Chem. A 2001, 105, 4042-4051.

(30) Zhang, J. X.; Hase, W. L. J. Phys. Chem. A 2010, 114, 9635-9643.

(31) O’Hair, R. A. J.; Davico, G. E.; Hacaloglu, J.; Dang, T. T.; DePuy, C. H.; Bierbaum, V. M. Journal of the American Chemical Society 1994, 116, 3609-3610.

(32) Xantheas, S.; Dunning Jr, T. J. Phys. Chem. 1994, 98, 13489-13497.

(33) Robertson, W.; Johnson, M. Annu. Rev. Phys. Chem. 2003, 54, 173-213.

(34) Roscioli, J. R.; Diken, E. G.; Johnson, M. A.; Horvath, S.; McCoy, A. B. J. Phys. Chem. A 2006, 110, 4943-4952.

(35) Li, G. S.; Hase, W. L. J. Am. Chem. Soc. 1999, 121, 7124-7129.

(36) Sun, L.; Hase, W. L. Rev. Comput. Chem. 2003, 19, 79-146.

(37) Townsend, D.; Lahankar, S. A.; Lee, S. K.; Chambreau, S. D.; Suits, A. G.; Zhang, X.; Rheinecker, J.; Harding, L. B.; Bowman, J. M. Science 2004, 306, 1158-1161.

(38) Bowman, J.; Shepler, B. Annu. Rev. Phys. Chem. 2011, 62, 531-553. 
(39) Syage, J. A. Chem. Phys. 1995, 207, 411-426.

(40) Eppink, A. T. J. B.; Parker, D. H. Rev. Sci. Instrum. 1997, 68, 3477-3484.

(41) Gebhardt, C. R.; Rakitzis, T. P.; Samartzis, P. C.; Ladopoulos, V.; Kitsopoulos, T. N. Rev. Sci. Instrum. 2001, 72, 3848-3853.

(42) Li, W.; Huang, C.; Patel, M.; Wilson, D.; Suits, A. J. Chem. Phys. 2006, 124, 011102.

(43) Lin, J. J.; Zhou, J.; Shiu, W.; Liu, K. Rev. Sci. Instrum. 2003, 74, 2495-2500.

(44) SIMION 7.0, Idaho National Engineering Laboratory. 1999.

(45) Dunning, Jr., T. H. J. Chem. Phys. 1989, 90, 1007-1023.

(46) Woon, D. E.; Dunning, Jr., T. H. J. Chem. Phys. 1993, 98, 1358-1371.

(47) Wadt, W. R.; Hay, P. J. J. Chem. Phys. 1985, 82, 284-298.

(48) Hu, W.; Truhlar, D. G. J. Phys. Chem. 1994, 98, 1049-1052.

(49) Hamprecht, F. A.; Cohen, A.; Tozer, D. J.; Handy, N. C. J. Chem. Phys. 1998, 109, 62646271.

(50) Raghavachari, K.; Trucks, G. W.; Pople, J. A.; Headgordon, M. Chem. Phys. Lett. 1989, 157, 479-483.

(51) Lieder, C. A.; Brauman, J. I. J. Am. Chem. Soc. 1974, 96, 4028-4030.

(52) Peslherbe, G.; Wang, H.; Hase, W. Adv. Chem. Phys. 1999, 105, 171-201.

(53) Hu, X.; Hase, W.; Pirraglia, T. J. Comp. Chem. 1991, 12, 1014-1024.

(54) Hase, W. L.; Duchovic, R. J.; Hu, X.; Komornicki, A.; Lim, K. F.; Lu, D. H.; Peslherbe, G. H.; Swamy, K. N.; Vande Linde, S. R.; Varandas, A.; Wang, H.; Wolf, R. J. Quant. Chem. Prog. Ex. 1996, 16, 671 . 
(55) Kendall, R. A.; Apra, E.; Bernholdt, D. E.; Bylaska, E. J.; Dupuis, M.; Fann, G. I.; Harrison, R. J.; Ju, J.; Nichols, J. A.; Nieplocha, J.; Straatsma, T. P.; Windus, T. L.; Wong, A. T. Comput. Phys. Commun. 2000, 128, 260-283.

(56) Valiev, M.; Bylaska, E.; Govind, N.; Kowalski, K.; Straatsma, T.; Van Dam, H.; Wang, D.; Nieplocha, J.; Apra, E.; Windus, T.; de Jong, W. A. Comput. Phys. Commun. 2010, 181, $1477-1489$.

(57) Vande Linde, S. R.; Hase, W. L. J. Am. Chem. Soc. 1989, 111, 2349-2351.

(58) Mann, D. J.; Hase, W. L. J. Phys. Chem. A 1998, 102, 6208-6214.

(59) Wang, Y.; Hase, W. L.; Wang, H. J. Chem. Phys. 2003, 118, 2688-2695.

(60) Glukhovtsev, M. N.; Pross, A.; Radom, L. J. Am. Chem. Soc. 1996, 118, 6273-6284.

(61) Schmatz, S.; Botschwina, P.; Stoll, H. Int. J. Mass Spectrom. 2000, 201, 277-282.

(62) Su, T.; Wang, H. B.; Hase, W. L. J. Phys. Chem. 1998, 102, 9819-9828.

(63) Wang, H.; Peslherbe, G. H.; Hase, W. L. J. Am. Chem. Soc. 1994, 116, 9644-9651.

(64) Tachikawa, H.; Igarashi, M. Chem. Phys. Lett. 1999, 303, 81-86.

(65) Zhang, W.; Kawamata, H.; Liu, K. Science 2009, 325, 303-306.

(66) Czakó, G.; Bowman, J. J. A. Chem. Soc. 2009, 131, 17534-17535.

(67) Su, T. J. Chem. Phys. 1994, 100, 4703-4703.

(68) Vande Linde, S. R.; Hase, W. L. J. Chem. Phys. 1990, 93, 7962-7980. 


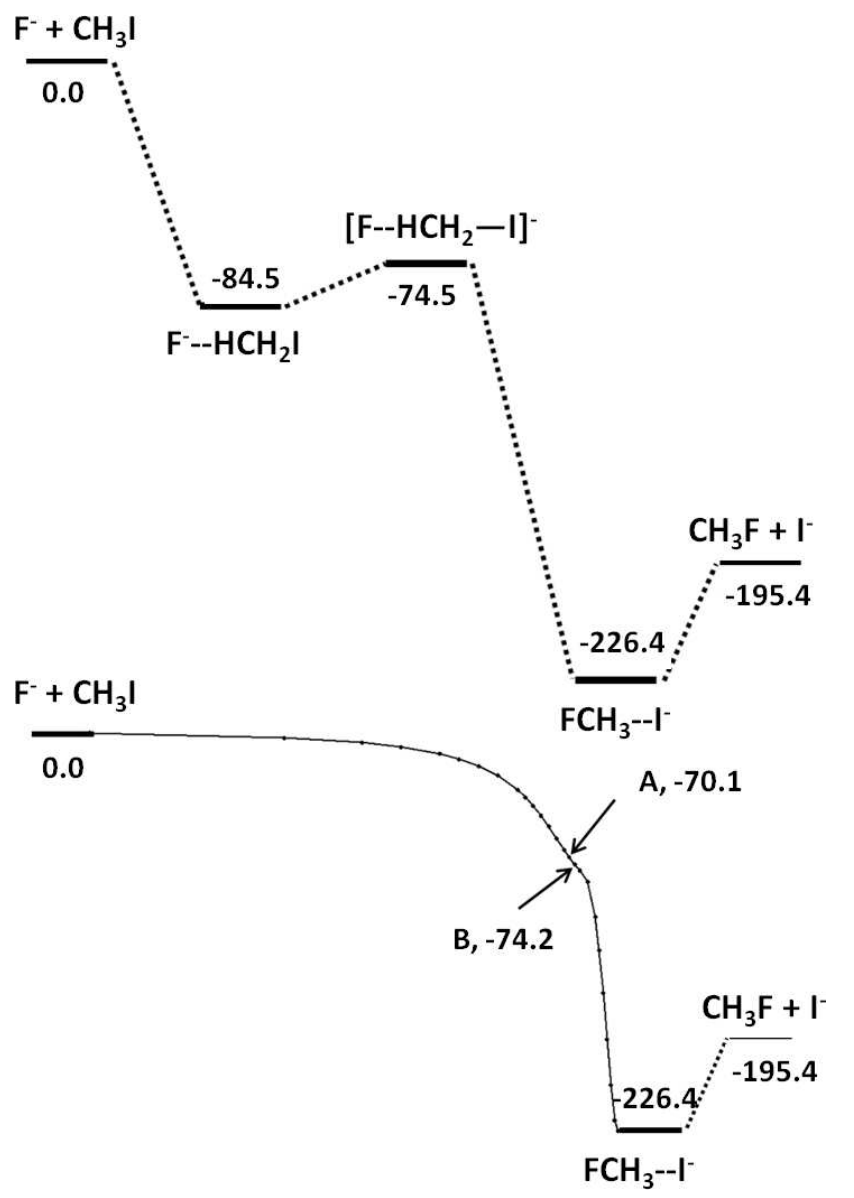

Figure 1: The intrinsic reaction coordinate (IRC) potential energy curve for the $\mathrm{F}^{-}+\mathrm{CH}_{3} \mathrm{I} \rightarrow$ $\mathrm{CH}_{3} \mathrm{~F}+\mathrm{I}^{-}$reaction ${ }^{30}$ and the potential energy curve with the reactive system constrained to a $\mathrm{C}_{3 v}$ geometry of the reactive system optimized as the $\mathrm{F}-\mathrm{C}$ distance is varied. Point $\mathrm{A}$ has a $\mathrm{C}-\mathrm{F}$ distance equal to that for the pre-reaction complex on the IRC potential energy curve and point $\mathrm{B}$ has a $\mathrm{C}-\mathrm{F}$ distance equal to that for the TS on the IRC potential energy curve. For the $\mathrm{C}_{3 v}$ curve, the only minimum/maximum is the post-reaction ion-dipole complex. Calculations are performed at the DFT/B97-1/ECP/d level of theory ${ }^{30}$ and energies are in $\mathrm{kJ} / \mathrm{mol}$. 


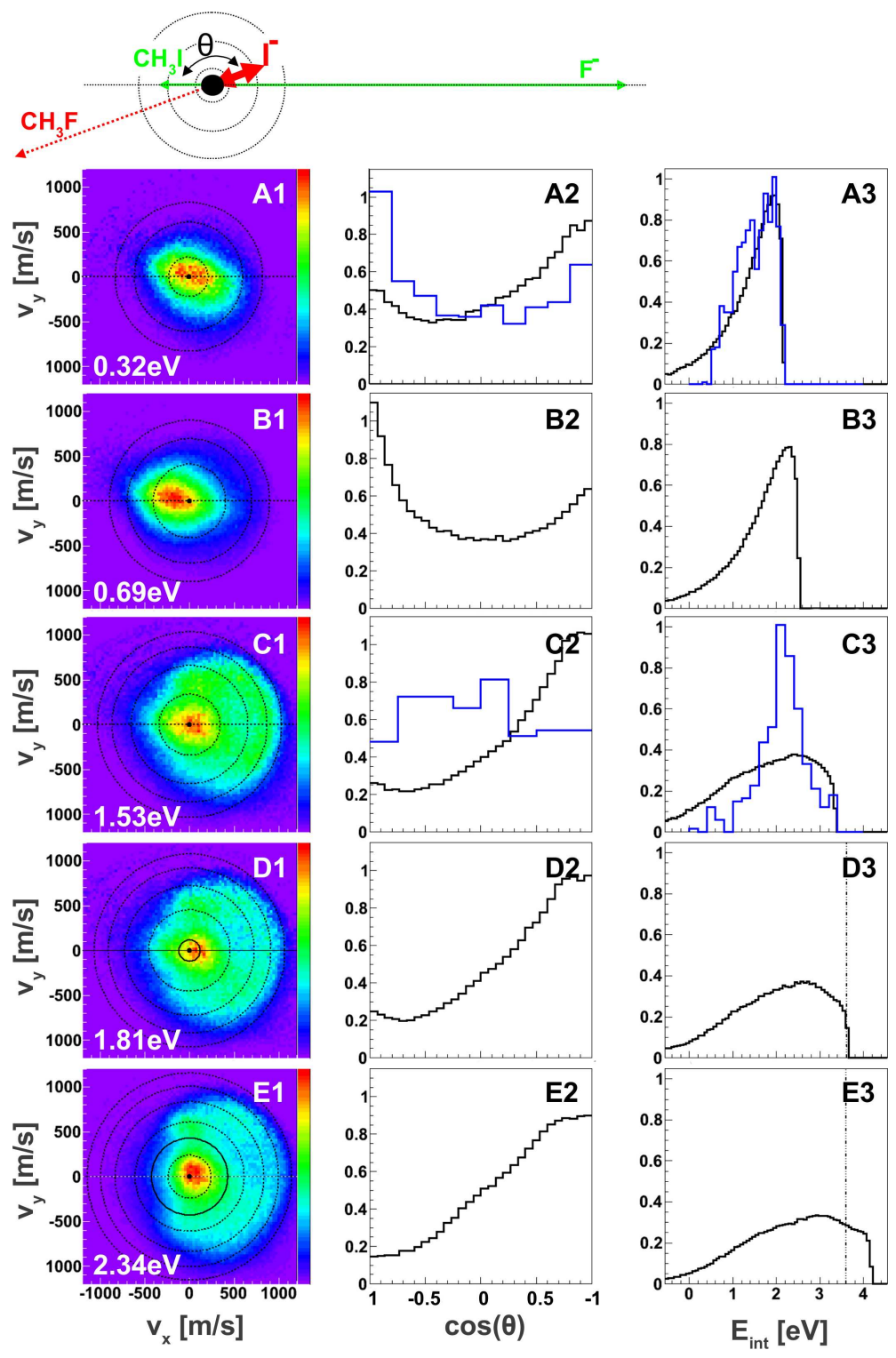

Figure 2: A1-E1: Measured velocity images of the $\mathrm{I}^{-}$product from reactive scattering of $\mathrm{F}^{-}$ and $\mathrm{CH}_{3} \mathrm{I}$ at different collision energies (see the schematic Newton diagram in the center-of-mass frame at the top). The dashed ring with the largest radius in each image represents the kinematical cutoff for the $S_{N} 2$ reaction with no product excitation. The smaller rings represent spheres of increasing product internal excitation, spaced at $1 \mathrm{eV}$ intervals. The solid circle and the vertical line in panels E1 and E3 indicate the threshold energy for breakup into $\mathrm{CH}_{2}+\mathrm{HF}$. A2-E2 and A3E3: Histograms of the scattering angle and the internal excitation for the same relative collision energies (black lines). The $E_{\text {int }}$-histograms show a sharp upper bound, caused by products with vanishing velocity and a more diffuse lower bound, due to the finite energy resolution. Simulation results for 0.32 and $1.53 \mathrm{eV}$ are shown in blue. 

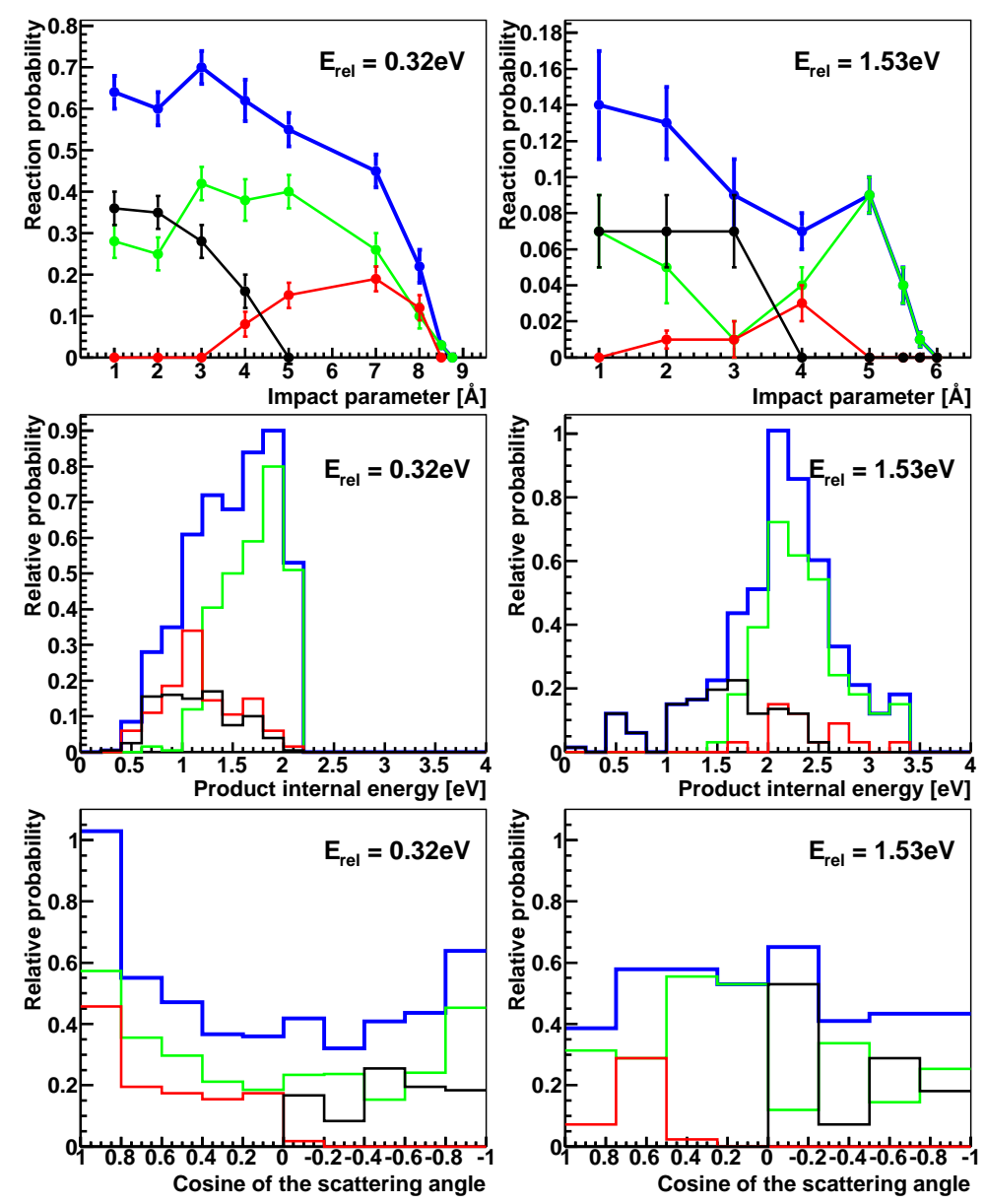

Figure 3: Trajectory simulation results for the $\mathrm{F}^{-}+\mathrm{CH}_{3} \mathrm{I} \rightarrow \mathrm{FCH}_{3}+\mathrm{I}^{-}$reaction at 0.32 and $1.53 \mathrm{eV}$ collision energies. The two top panels show the reaction probability versus impact parameter for the three identified atomic-level reaction mechanisms and for the total reaction: direct rebound (black), direct stripping (red), indirect (green), total (blue). The product scattering angle and internal energy distributions are given in the center and bottom panels for the direct rebound (black), the direct stripping (red), and the indirect (green) atomic-level mechanisms, as well as for the total scattering (blue). 
Table 1: Measured average internal energies $\bar{E}_{\text {int }}$ for the $\mathrm{CH}_{3} \mathrm{~F}$ product as a function of relative collision energy. The third column shows the estimated average accuracy of the internal energy and the fourth column shows the average internal energies corrected for experimental broadening (see text for details). The values in square brackets show the fraction of internal energy with respect to the total available energy with the values in round brackets denoting the estimated accuracies on the last digit. The values in bold may be compared with the simulation results in table 2 .

\begin{tabular}{llll}
\hline$E_{\text {rel }}[\mathrm{eV}]$ & $\bar{E}_{\text {int }}[\mathrm{eV}]$ & $\bar{\sigma}_{\text {int }}$ & $\bar{E}_{\text {int }}^{\text {corr }}[\mathrm{eV}]$ \\
\hline 0.32 & $1.36[0.63]$ & 0.20 & $\mathbf{1 . 5 2}[\mathbf{0 . 7 0}(\mathbf{9})]$ \\
0.69 & $1.62[0.64]$ & 0.22 & $1.80[0.71(9)]$ \\
1.53 & $1.77[0.53]$ & 0.28 & $\mathbf{1 . 9 9}[\mathbf{0 . 5 9 ( 8 )}]$ \\
1.81 & $1.99[0.54]$ & 0.33 & $2.25[0.62(9)]$ \\
2.34 & $2.36[0.57]$ & 0.27 & $2.58[0.62(7)]$ \\
\hline
\end{tabular}

Table 2: Simulation of the average fractions of $\mathrm{F}^{-}+\mathrm{CH}_{3} \mathrm{I} \rightarrow \mathrm{CH}_{3} \mathrm{~F}+\mathrm{I}^{-}$product energy partitioning. The f's are fractions of energy partitioning for rotational, vibrational, relative translational, and internal energy. The total is the combined energy partitioning for these mechanisms, the values in bold face may be compared with the experimental results in table 1.

\begin{tabular}{lcccc}
\hline & $f_{\text {rot }}^{\prime}$ & $f_{\text {vib }}^{\prime}$ & $f_{\text {rel }}^{\prime}$ & $f_{\text {int }}^{\prime}$ \\
\hline Direct rebound & $0.07(1)$ & $E_{\text {rel }}=0.32 \mathrm{eV}$ & & \\
Direct stripping & $0.05(1)$ & $0.50(4)$ & $0.46(3)$ & $0.54(3)$ \\
Indirect & $0.08(1)$ & $0.71(2)$ & $0.45(4)$ & $0.55(4)$ \\
Total & $0.07(1)$ & $0.62(2)$ & $0.31(2)$ & $0.79(2)$ \\
& & $\mathbf{0 . 6 9}(2)$ \\
Direct rebound & $0.19(3)$ & $E_{\text {rel }}=1.53 \mathrm{eV}$ & & \\
Direct stripping & $0.27(3)$ & $0.45(4)$ & $0.54(3)$ & $0.46(3)$ \\
Indirect & $0.07(3)$ & $0.62(4)$ & $0.31(2)$ & $0.72(2)$ \\
Total & $0.12(3)$ & $0.51(6)$ & $0.69(2)$ \\
\hline
\end{tabular}




\section{Graphical TOC Entry}

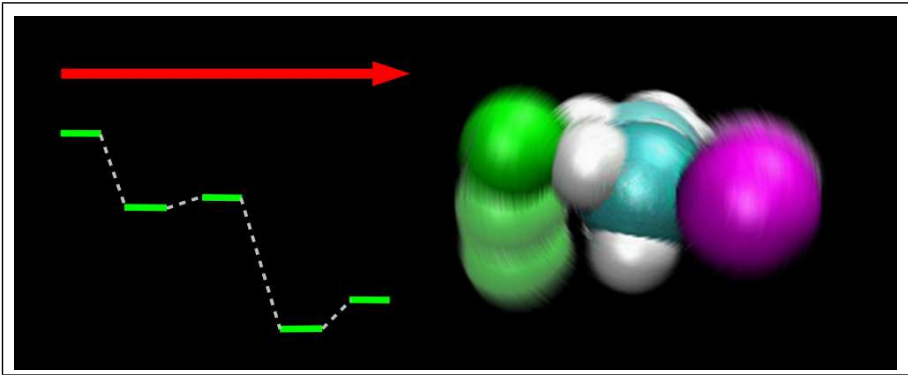

Table of Contents (TOC) graphic. 